\title{
Tracking Moving Acoustic Sources with a Network of Sensors
}

\author{
Richard J. Kozick ${ }^{a}$ and Brian M. Sadler ${ }^{b}$ \\ ${ }^{a}$ Bucknell University, Dept. of Electrical Engineering, Lewisburg, PA 17837 \\ ${ }^{b}$ Army Research Laboratory, AMSRL-CI-CN, 2800 Powder Mill Road, Adelphi, MD 20783
}

\begin{abstract}
This paper presents issues and algorithms for the problem of source tracking with a network of aeroacoustic sensors. We study fusion of data from sensors that are widely separated, and we give particular attention to the important issues of limited communication bandwidth between sensor nodes, effects of source motion, coherence loss between signals measured at different sensors, signal bandwidth, and noise. We compare the tracking performance of various schemes, including joint (coherent) processing of all sensor data, as well as datareduction schemes that employ distributed computation and reduced communication bandwidth with a fusion center. The key result of our analysis is a quantification of the potential gain in source tracking accuracy that is achievable with greater communication bandwidth and joint processing of sensor data. We show that the potential gain in accuracy depends critically on the scenario, as determined by the source motion parameters, signal coherence between sensors, bandwidth of the source signals, and noise level. For scenarios that admit increased accuracy with joint processing, we present a bandwidth-efficient algorithm that involves beamforming at small-aperture sensor arrays combined with time-delay estimation between widely-spaced sensor arrays.
\end{abstract}

Keywords: Aeroacoustic sensor arrays, source localization and tracking, imperfect spatial coherence, decentralized signal processing, data compression and fusion.

\section{INTRODUCTION}

We are concerned with tracking moving sources using a network of aeroacoustic sensors. We assume that the sensors are placed in an "array of arrays" configuration containing several small-aperture arrays distributed over a wide area. Each array contains local processing capability and a communication link with a fusion center. A standard approach for estimating the source locations involves bearing estimation at the individual arrays, communication of the bearings to the fusion center, and processing of the bearing estimates at the fusion center with a tracking algorithm. ${ }^{1-5}$ This approach is characterized by low communication bandwidth and low complexity, but the localization accuracy may be inferior to the optimal solution in which the fusion center jointly processes all of the sensor data. The optimal solution requires high communication bandwidth and high processing complexity. The amount of improvement in localization accuracy that is enabled by greater communication bandwidth and processing complexity is dependent on the scenario, which we characterize in terms of the source motion parameters, the power spectra (and bandwidth) of the signals and noise in the sensor data, the coherence between the source signals received at widely separated sensors, and the observation time (amount of data). We present a framework in this paper to identify scenarios that have the potential for improved localization accuracy relative to the standard bearings-only tracking method. We propose an algorithm that is bandwidth-efficient and nearly optimal that uses beamforming at small-aperture sensor arrays and time-delay estimation between widely-separated sensors.

The sensor signals are modeled as Gaussian random processes, which allows deterministic as well as random propagation effects to be included. Our previous work $^{6,7}$ considered a single source with fixed position (no motion). We extend the analysis in this paper to moving sources that follow a parametric motion model.

This paper is organized as follows. The sensor data model is presented in Section 2 for the case of a nonmoving source. Results on time-delay estimation with partially-coherent signals are presented in Section 3, which summarizes and extends our previous work. ${ }^{7}$ The sensor data model is extended to moving sources in Section 4. An algorithm is outlined in Section 5, and concluding remarks are given in Section 6.

Author email: kozick@bucknell.edu and bsadler@arl.army.mil 


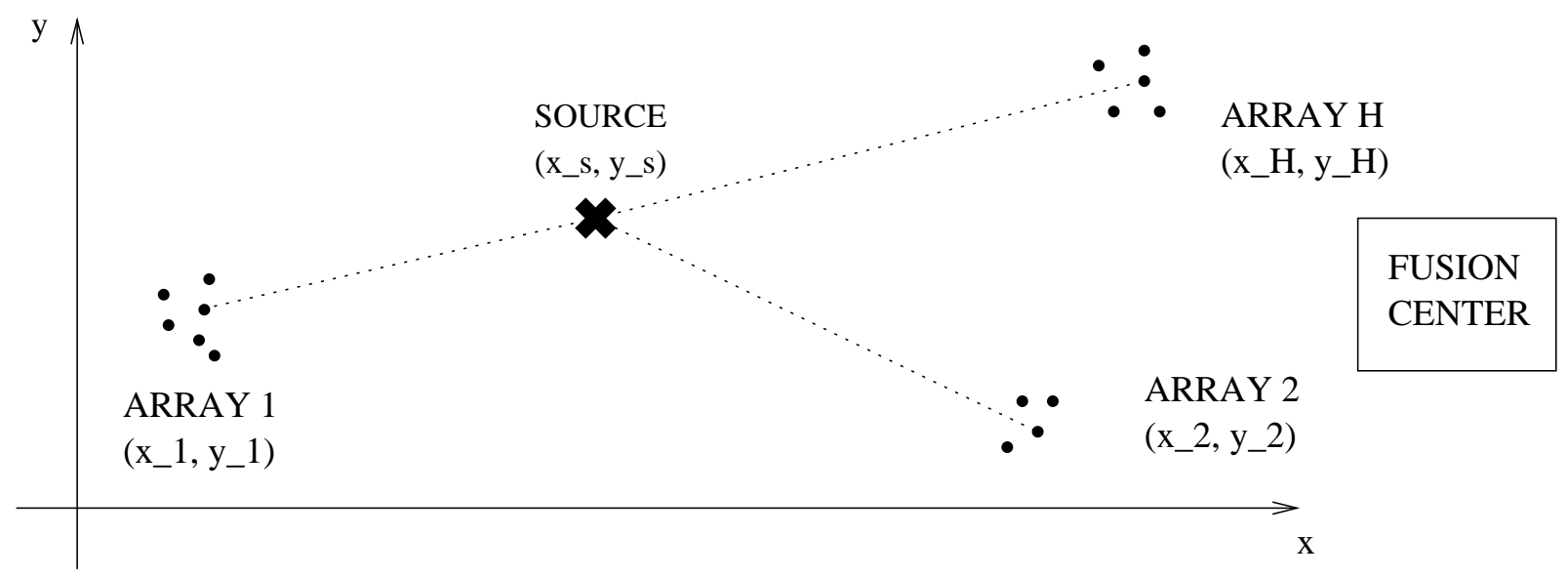

Figure 1. Geometry of non-moving source location and an array of arrays. A communication link is available between each array and the fusion center.

\section{DATA MODEL FOR A NON-MOVING SOURCE}

A model is formulated in this section for the discrete-time signals received by the sensors in an array of arrays. To begin, suppose a single non-moving source is located at coordinates $\left(x_{s}, y_{s}\right)$ in the $(x, y)$ plane, and consider $H$ arrays that are distributed in the same plane, as illustrated in Figure 1. Each array $h \in\{1, \ldots, H\}$ contains $N_{h}$ sensors and has a reference sensor located at coordinates $\left(x_{h}, y_{h}\right)$. The location of sensor $n \in\left\{1, \ldots, N_{h}\right\}$ is at $\left(x_{h}+\Delta x_{h n}, y_{h}+\Delta y_{h n}\right)$, where $\left(\Delta x_{h n}, \Delta y_{h n}\right)$ is the relative location with respect to the reference sensor. If $c$ is the speed of propagation, then the propagation time from the source to the reference sensor on array $h$ is

$$
\tau_{h}=\frac{d_{h}}{c}=\frac{1}{c}\left[\left(x_{s}-x_{h}\right)^{2}+\left(y_{s}-y_{h}\right)^{2}\right]^{1 / 2},
$$

where $d_{h}$ is the distance from the source to array $h$. We model the wavefronts over individual array apertures as perfectly coherent plane waves. Then in the far-field approximation, the propagation time from the source to sensor $n$ on array $h$ is expressed by $\tau_{h}+\tau_{h n}$, where

$$
\tau_{h n} \approx-\frac{1}{c}\left[\frac{x_{s}-x_{h}}{d_{h}} \Delta x_{h n}+\frac{y_{s}-y_{h}}{d_{h}} \Delta y_{h n}\right]=-\frac{1}{c}\left[\left(\cos \phi_{h}\right) \Delta x_{h n}+\left(\sin \phi_{h}\right) \Delta y_{h n}\right]
$$

is the propagation time from the reference sensor on array $h$ to sensor $n$ on array $h$, and $\phi_{h}$ is the bearing of the source with respect to array $h$. Note that while the far-field approximation (2) is reasonable over individual array apertures, the wavefront curvature that is inherent in (1) must be retained in order to model wide separations between arrays.

The time signal received at sensor $n$ on array $h$ due to the source will be represented as $s_{h}\left(t-\tau_{h}-\tau_{h n}\right)$, where the vector of signals $\mathbf{s}(t)=\left[s_{1}(t), \ldots, s_{H}(t)\right]^{T}$ received at the $H$ arrays are modeled as real-valued, continuoustime, zero-mean, jointly wide-sense stationary, Gaussian random processes with $-\infty<t<\infty$. These processes are fully specified by the $H \times H$ cross-correlation function matrix

$$
\mathbf{R}_{s}(\tau)=E\left\{\mathbf{s}(t+\tau) \mathbf{s}(t)^{T}\right\}
$$

where $E$ denotes expectation, superscript $T$ denotes transpose, and we will later use the notation superscript $*$ and superscript $H$ to denote complex conjugate and conjugate transpose, respectively. The $(g, h)$ element in $(3)$ is the cross-correlation function

$$
r_{s, g h}(\tau)=E\left\{s_{g}(t+\tau) s_{h}(t)\right\}
$$

between the signals received at arrays $g$ and $h$. The correlation functions (3) and (4) are equivalently characterized by their Fourier transforms, which are the cross-spectral density functions

$$
G_{s, g h}(\omega)=\mathcal{F}\left\{r_{s, g h}(\tau)\right\}=\int_{-\infty}^{\infty} r_{s, g h}(\tau) \exp (-j \omega \tau) d \tau
$$


and the associated cross-spectral density matrix

$$
\mathbf{G}_{s}(\omega)=\mathcal{F}\left\{\mathbf{R}_{s}(\tau)\right\} .
$$

The diagonal elements $G_{s, h h}(\omega)$ of (6) are the power spectral density (PSD) functions of the signals $s_{h}(t)$, and hence they describe the distribution of average signal power with frequency. The model allows the PSD to vary from one array to another to reflect propagation differences and source aspect angle differences.

The off-diagonal elements of (6), $G_{s, g h}(\omega)$, are the cross-spectral density (CSD) functions for the signals $s_{g}(t)$ and $s_{h}(t)$ received at distinct arrays $g \neq h$. In general, the CSD functions have the form

$$
G_{s, g h}(\omega)=\gamma_{s, g h}(\omega)\left[G_{s, g g}(\omega) G_{s, h h}(\omega)\right]^{1 / 2},
$$

where $\gamma_{s, g h}(\omega)$ is the spectral coherence function for the signals, which has the property $0 \leq\left|\gamma_{s, g h}(\omega)\right| \leq 1$. Coherence magnitude $\left|\gamma_{s, g h}(\omega)\right|=1$ corresponds to perfect correlation between the signals at sensors $g$ and $h$, while the partially coherent case $\left|\gamma_{s, g h}(\omega)\right|<1$ models random effects in the propagation paths from the source to sensors $g$ and $h$. Note that our assumption of perfect spatial coherence across individual arrays implies that the random propagation effects have negligible impact on the intra-array delays $\tau_{h n}$ in (2) and the bearings $\phi_{1}, \ldots \phi_{H}$.

The signal received at sensor $n$ on array $h$ is modeled as a sum of the delayed source signal and noise,

$$
z_{h n}(t)=s_{h}\left(t-\tau_{h}-\tau_{h n}\right)+w_{h n}(t),
$$

where the noise signals $w_{h n}(t)$ are modeled as real-valued, continuous-time, zero-mean, jointly wide-sense stationary, Gaussian random processes that are mutually uncorrelated at distinct sensors, and are uncorrelated from the signals. That is, the noise correlation properties are

$$
\begin{aligned}
E\left\{w_{g m}(t+\tau) w_{h n}(t)\right\} & =r_{w}(\tau) \delta_{g h} \delta_{m n} \\
E\left\{w_{g m}(t+\tau) s_{h}(t)\right\} & =0
\end{aligned}
$$

where $r_{w}(\tau)$ is the noise autocorrelation function, and the noise PSD is $G_{w}(\omega)=\mathcal{F}\left\{r_{w}(\tau)\right\}$. We then collect the observations at each array $h$ into $N_{h} \times 1$ vectors $\mathbf{z}_{h}(t)=\left[z_{h 1}(t), \ldots, z_{h, N_{h}}(t)\right]^{T}$ for $h=1, \ldots, H$, and we further collect the observations from the $H$ arrays into a $\left(N_{1}+\cdots+N_{H}\right) \times 1$ vector

$$
\mathbf{Z}(t)=\left[\begin{array}{c}
\mathbf{z}_{1}(t) \\
\vdots \\
\mathbf{z}_{H}(t)
\end{array}\right]
$$

The elements of $\mathbf{Z}(t)$ in (11) are zero-mean, jointly wide-sense stationary, Gaussian random processes. We can express the CSD matrix of $\mathbf{Z}(t)$ in a convenient form with the following definitions. The array manifold for array $h$ at frequency $\omega$ is

$$
\mathbf{a}_{h}(\omega)=\left[\begin{array}{c}
\exp \left(-j \omega \tau_{h 1}\right) \\
\vdots \\
\exp \left(-j \omega \tau_{h, N_{h}}\right)
\end{array}\right]=\left[\begin{array}{c}
\exp \left[j \frac{\omega}{c}\left(\left(\cos \phi_{h}\right) \Delta x_{h 1}+\left(\sin \phi_{h}\right) \Delta y_{h 1}\right)\right] \\
\vdots \\
\exp \left[j \frac{\omega}{c}\left(\left(\cos \phi_{h}\right) \Delta x_{h, N_{h}}+\left(\sin \phi_{h}\right) \Delta y_{h, N_{h}}\right)\right]
\end{array}\right],
$$

using $\tau_{h n}$ from (2) and assuming that the sensors have omnidirectional response to sources in the plane of the array. Let us define the relative time delay of the signal at arrays $g$ and $h$ as

$$
D_{g h}=\tau_{g}-\tau_{h},
$$

where $\tau_{h}$ is defined in (1). Then the cross-spectral density matrix of $\mathbf{Z}(t)$ in (11) has the form

$$
\mathbf{G}_{\mathbf{Z}}(\omega)=
$$




$$
\left[\begin{array}{ccc}
\mathbf{a}_{1}(\omega) \mathbf{a}_{1}(\omega)^{H} G_{s, 11}(\omega) & \cdots & \mathbf{a}_{1}(\omega) \mathbf{a}_{H}(\omega)^{H} \exp \left(-j \omega D_{1 H}\right) G_{s, 1 H}(\omega) \\
\vdots & \ddots & \vdots \\
\mathbf{a}_{H}(\omega) \mathbf{a}_{1}(\omega)^{H} \exp \left(+j \omega D_{1 H}\right) G_{s, 1 H}(\omega)^{*} & \cdots & \mathbf{a}_{H}(\omega) \mathbf{a}_{H}(\omega)^{H} G_{s, H H}(\omega)
\end{array}\right]+G_{w}(\omega) \mathbf{I} .
$$

The source CSD functions $G_{s, g h}(\omega)$ in (14) can be expressed in terms of the signal spectral coherence $\gamma_{s, g h}(\omega)$ using (7). Note that (14) depends on the source location parameters $\left(x_{s}, y_{s}\right)$ through the bearings $\phi_{h}$ in $\mathbf{a}_{h}(\omega)$ and the pairwise time-delay differences $D_{g h}$.

\subsection{Cramér-Rao Bound (CRB)}

The Cramér-Rao bound (CRB) provides a lower bound on the variance of any unbiased estimator. The problem of interest is estimation of the source location parameter vector $\boldsymbol{\Theta}=\left[x_{s}, y_{s}\right]^{T}$ using $T$ samples of the sensor signals $\mathbf{Z}(0), \mathbf{Z}\left(T_{s}\right), \ldots, \mathbf{Z}\left((T-1) \cdot T_{s}\right)$, where $T_{s}$ is the sampling period. The total observation time is $\mathcal{T}=T \cdot T_{s}$. Let us denote the sampling rate by $f_{s}=1 / T_{s}$ and $\omega_{s}=2 \pi f_{s}$. We will assume that the continuous-time random processes $\mathbf{Z}(t)$ are band-limited, and that the sampling rate $f_{s}$ is greater than twice the bandwidth of the processes. Then Friedlander ${ }^{8,9}$ has shown that the Fisher information matrix (FIM) $\mathbf{J}$ for the parameters $\Theta$ based on the samples $\mathbf{Z}(0), \mathbf{Z}\left(T_{s}\right), \ldots, \mathbf{Z}\left((T-1) \cdot T_{s}\right)$ has elements

$$
J_{i j}=\frac{\mathcal{T}}{4 \pi} \int_{0}^{\omega_{s}} \operatorname{tr}\left\{\frac{\partial \mathbf{G}_{\mathbf{Z}}(\omega)}{\partial \theta_{i}} \mathbf{G}_{\mathbf{Z}}(\omega)^{-1} \frac{\partial \mathbf{G}_{\mathbf{Z}}(\omega)}{\partial \theta_{j}} \mathbf{G}_{\mathbf{Z}}(\omega)^{-1}\right\} d \omega, \quad i, j=1,2,
$$

where "tr" denotes the trace of the matrix. The CRB matrix $\mathbf{C}=\mathbf{J}^{-1}$ then has the property that the covariance matrix of any unbiased estimator $\hat{\boldsymbol{\Theta}}$ satisfies $\operatorname{Cov}(\hat{\boldsymbol{\Theta}})-\mathbf{C} \geq \mathbf{0}$, where $\geq \mathbf{0}$ means that $\operatorname{Cov}(\hat{\boldsymbol{\Theta}})-\mathbf{C}$ is positive semidefinite. ${ }^{10}$ Equation (15) provides a convenient way to compute the FIM for the array of arrays model as a function of the signal coherence between distributed arrays, the signal and noise bandwidth and power spectra, and the sensor placement geometry. The CRB is evaluated for various scenarios in our previous works. ${ }^{6,7}$

\section{TIME-DELAY ESTIMATION (TDE)}

Let us parameterize the model in (14) by the bearings $\phi_{h}$ and the time-delay differences $D_{g h}$. Then we must address the issue of time-delay estimation with signals that are partially coherent when $\left|\gamma_{s, g h}\right|<1$. We consider this problem first for the case of $H=2$ sensors, as illustrated in Figure 2a with the differential time delay defined as $D=D_{21}$. It follows from (14) that the CSD matrix of the sensor data in Figure 2a is

$$
\begin{aligned}
\operatorname{CSD}\left[\begin{array}{l}
z_{1}(t) \\
z_{2}(t)
\end{array}\right] & =\mathbf{G}_{\mathbf{Z}}(\omega)= \\
& {\left[\begin{array}{cc}
G_{s, 11}(\omega)+G_{w}(\omega) & e^{+j \omega D} \gamma_{s, 12}(\omega)\left[G_{s, 11}(\omega) G_{s, 22}(\omega)\right]^{1 / 2} \\
e^{-j \omega D} \gamma_{s, 12}(\omega)^{*}\left[G_{s, 11}(\omega) G_{s, 22}(\omega)\right]^{1 / 2} & G_{s, 22}(\omega)+G_{w}(\omega)
\end{array}\right] . }
\end{aligned}
$$

The signal coherence function $\gamma_{s, 12}(\omega)$ describes the degree of correlation that remains in the signal emitted by the source at each frequency $\omega$ after propagating to sensors 1 and 2. Next, we develop an SNR-like expression for the two-sensor case that appears in all subsequent expressions for fundamental limits on TD and Doppler estimation. We begin with the magnitude-squared coherence ${ }^{11}$ (MSC) of the observed signals $z_{1}(t), z_{2}(t)$ as a function of the signal coherence magnitude, $\left|\gamma_{s, 12}(\omega)\right|$, and other spectral density parameters:

$$
\begin{aligned}
\operatorname{MSC}_{z}\left(\left|\gamma_{s, 12}(\omega)\right|\right) & =\frac{\left|\operatorname{CSD}\left[z_{1}(t), z_{2}(t)\right]\right|^{2}}{\operatorname{PSD}\left[z_{1}(t)\right] \cdot \operatorname{PSD}\left[z_{2}(t)\right]}=\frac{\left|\gamma_{s, 12}(\omega)\right|^{2} G_{s, 11}(\omega) G_{s, 22}(\omega)}{\left[G_{s, 11}(\omega)+G_{w}(\omega)\right]\left[G_{s, 22}(\omega)+G_{w}(\omega)\right]} \\
& =\frac{\left|\gamma_{s, 12}(\omega)\right|^{2}}{\left[1+\left(\frac{G_{s, 11}(\omega)}{G_{w}(\omega)}\right)^{-1}\right]\left[1+\left(\frac{G_{s, 22}(\omega)}{G_{w}(\omega)}\right)^{-1}\right]} \leq 1
\end{aligned}
$$


Then the following "SNR" expression appears in subsequent performance bounds:

$$
\begin{aligned}
\operatorname{SNR}\left(\left|\gamma_{s, 12}(\omega)\right|\right) & =\frac{\operatorname{MSC}_{z}\left(\left|\gamma_{s, 12}(\omega)\right|\right)}{1-\operatorname{MSC}_{z}\left(\left|\gamma_{s, 12}(\omega)\right|\right)} \\
& =\left\{\frac{1}{\left|\gamma_{s, 12}(\omega)\right|^{2}}\left[1+\left(\frac{G_{s, 11}(\omega)}{G_{w}(\omega)}\right)^{-1}\right]\left[1+\left(\frac{G_{s, 22}(\omega)}{G_{w}(\omega)}\right)^{-1}\right]-1\right\}^{-1} \\
& \leq \frac{\left|\gamma_{s, 12}(\omega)\right|^{2}}{1-\left|\gamma_{s, 12}(\omega)\right|^{2}}
\end{aligned}
$$

The inequality (20) shows that signal coherence loss $\left(\left|\gamma_{s, 12}(\omega)\right|<1\right)$ severely limits the "SNR" quantity that characterizes performance, even if the SNR per sensor $G_{s, i i}(\omega) / G_{w}(\omega)$ is very large.

In this section, we summarize and further study performance bounds on time-delay estimation (TDE) with partially coherent signals that were originally presented in our earlier work. ${ }^{7}$ We can use (16) in (15) to find the CRB for TDE with $H=2$ sensors, yielding

$$
\operatorname{CRB}(D)=\frac{4 \pi}{\mathcal{T}}\left[\int_{0}^{\omega_{s}} \omega^{2} \operatorname{SNR}\left(\left|\gamma_{s, 12}(\omega)\right|\right) d \omega\right]^{-1}
$$

where $\mathcal{T}$ is the total observation time of the sensor data and $\operatorname{SNR}\left(\left|\gamma_{s, 12}(\omega)\right|\right)$ is defined in (19). Let us consider the case in which the signal PSDs, the noise PSD, and the coherence are flat (constant) over a bandwidth $\Delta \omega$ $\mathrm{rad} / \mathrm{sec}$ centered at $\omega_{0} \mathrm{rad} / \mathrm{sec}$. If we omit the frequency dependence of $G_{s, 11}, G_{s, 22}, G_{w}$, and $\gamma_{s, 12}$, then the integral in (21) may be evaluated to yield the following CRB expression:

$$
\begin{aligned}
\operatorname{CRB}(D) & =\frac{1}{2 \omega_{0}^{2}\left(\frac{\Delta \omega \mathcal{T}}{2 \pi}\right)\left[1+\frac{1}{12}\left(\frac{\Delta \omega}{\omega_{0}}\right)^{2}\right] \operatorname{SNR}\left(\left|\gamma_{s, 12}\right|\right)} \\
& =\frac{1}{2 \omega_{0}^{2}\left(\frac{\Delta \omega \mathcal{T}}{2 \pi}\right)\left[1+\frac{1}{12}\left(\frac{\Delta \omega}{\omega_{0}}\right)^{2}\right]}\left\{\frac{1}{\left|\gamma_{s, 12}\right|^{2}}\left[1+\left(\frac{G_{s, 11}}{G_{w}}\right)^{-1}\right]\left[1+\left(\frac{G_{s, 22}}{G_{w}}\right)^{-1}\right]-1\right\} \\
& >\frac{1}{2 \omega_{0}^{2}\left(\frac{\Delta \omega \mathcal{T}}{2 \pi}\right)\left[1+\frac{1}{12}\left(\frac{\Delta \omega}{\omega_{0}}\right)^{2}\right]}\left[\frac{1}{\left|\gamma_{s, 12}\right|^{2}}-1\right] .
\end{aligned}
$$

The quantity $\left(\frac{\Delta \omega \cdot \mathcal{T}}{2 \pi}\right)$ is the time-bandwidth product of the observations, $\left(\frac{\Delta \omega}{\omega_{0}}\right)$ is the fractional bandwidth of the signal, and $G_{s, h h} / G_{w}$ is the SNR at sensor $h$. Note from the high-SNR limit in (24) that when the signals are partially coherent $\left|\gamma_{s, 12}\right|<1$, increased source power does not reduce the CRB. Improved TDE accuracy is obtained with partially coherent signals by increasing the observation time $\mathcal{T}$ or changing the spectral support of the signal, which is $\left[\omega_{0}-\Delta \omega / 2, \omega_{0}+\Delta \omega / 2\right]$. The spectral support of the signal is not controllable in passive TDE applications, so increased observation time is the only means for improving the TDE accuracy with partially coherent signals. Source motion becomes more important during long observation times, and in Section 4 we extend the model to include source motion.

The CRB in (23) agrees with known results for perfectly coherent signals ${ }^{11}$ and with results from the medical ultrasound literature ${ }^{12,13}$ for partially correlated speckle signals. The medical ultrasound application is distinguished from the aeroacoustic tracking of ground vehicles in that the former is typically an active system while the latter is passive. The medical ultrasound application therefore allows much more control over the SNR and bandwidth of the signals. In passive aeroacoustics, the received signals are emitted by a vehicle and are not controllable for the purposes of TD estimation.

With perfectly coherent signals, it is well-known that the CRB on TDE is achievable only when the SNR expression in (19) (with $\left|\gamma_{s, 12}(\omega)\right|=1$ ) exceeds a threshold. ${ }^{14,15}$ Next we show that for TDE with partially 
coherent signals, a similar threshold phenomenon occurs with respect to coherence. That is, the coherence must exceed a threshold in order to achieve the CRB (21) on TDE. We state the threshold coherence formula for the following simplified scenario. The signal and noise spectra are flat over a bandwidth of $\Delta \omega \mathrm{rad} / \mathrm{sec}$ centered at $\omega_{0} \mathrm{rad} / \mathrm{sec}$, and the observation time is $\mathcal{T}$ seconds. Further, assume that the signal PSDs are identical at each sensor, and define the following constants for notational simplicity:

$$
G_{s, 11}\left(\omega_{0}\right)=G_{s, 22}\left(\omega_{0}\right)=G_{s}, G_{w}\left(\omega_{0}\right)=G_{w} \text {, and } \gamma_{s, 12}\left(\omega_{0}\right)=\gamma_{s} .
$$

Then the SNR expression in (19) has the form

$$
\operatorname{SNR}\left(\left|\gamma_{s}\right|\right)=\left[\frac{1}{\left|\gamma_{s}\right|^{2}}\left(1+\frac{1}{\left(G_{s} / G_{w}\right)}\right)^{2}-1\right]^{-1} .
$$

The Ziv-Zakai bound developed by Weiss and Weinstein ${ }^{14,15}$ shows that the threshold SNR for CRB attainability is a function of the time-bandwidth product $\left(\frac{\Delta \omega \cdot \mathcal{T}}{2 \pi}\right)$ and the fractional bandwidth $\left(\frac{\Delta \omega}{\omega_{0}}\right)$,

$$
\mathrm{SNR}_{\text {thresh }}=\frac{6}{\pi^{2}\left(\frac{\Delta \omega \mathcal{T}}{2 \pi}\right)}\left(\frac{\omega_{0}}{\Delta \omega}\right)^{2}\left[\varphi^{-1}\left(\frac{1}{24}\left(\frac{\Delta \omega}{\omega_{0}}\right)^{2}\right)\right]^{2}
$$

where $\varphi(y)=1 / \sqrt{2 \pi} \int_{y}^{\infty} \exp \left(-t^{2} / 2\right) d t$. It follows that the threshold coherence value is

$$
\left|\gamma_{s}\right|^{2} \geq \frac{\left(1+\frac{1}{\left(G_{s} / G_{w}\right)}\right)^{2}}{1+\frac{1}{\mathrm{SNR}_{\text {thresh }}}}, \quad \text { so } \quad\left|\gamma_{s}\right|^{2} \geq \frac{1}{1+\frac{1}{\mathrm{SNR}_{\text {thresh }}}} \text { as } \quad \frac{G_{s}}{G_{w}} \rightarrow \infty .
$$

For a specific TDE scenario, the threshold SNR for CRB attainability is given by (27), and (28) provides a corresponding threshold coherence. Since $\left|\gamma_{s}\right|^{2} \leq 1$, (28) is useful only if $G_{s} / G_{w}>\mathrm{SNR}_{\text {thresh }}$.

Figures $2 \mathrm{~b}$, c, and d contain plots of the threshold coherence in (28) as a function of the time-bandwidth product $\left(\frac{\Delta \omega \cdot \mathcal{T}}{2 \pi}\right)$, SNR $\frac{G_{s}}{G_{w}}$, and fractional bandwidth $\left(\frac{\Delta \omega}{\omega_{0}}\right)$. Note that $\frac{G_{s}}{G_{w}}=10 \mathrm{~dB}$ is nearly equivalent to $\frac{G_{s}}{G_{w}} \rightarrow \infty$. We note that very large time-bandwidth product is required to overcome coherence loss when the fractional bandwidth is small at 0.1 . The variation of threshold coherence with fractional bandwidth is illustrated in Figure 2e. For a fixed threshold coherence value, such as 0.7, each doubling of the fractional bandwidth reduces the required time-bandwidth product by about a factor of 10 . We have presented computer simulation examples elsewhere $^{16}$ that verify the CRB and threshold coherence analysis for TDE.

Let us examine a narrowband signal scenario that is typical in aeroacoustics, with center frequency $f_{o}=$ $\omega_{o} /(2 \pi)=50 \mathrm{~Hz}$ and bandwidth $\Delta f=\Delta \omega /(2 \pi)=5 \mathrm{~Hz}$, so the fractional bandwidth is $\Delta f / f_{o}=0.1$. From Figure $2 \mathrm{~b}$, coherence $\left|\gamma_{s}\right|=0.8$ requires time-bandwidth product $\Delta f \cdot \mathcal{T}>200$, so the necessary time duration $\mathcal{T}=40 \mathrm{sec}$ for TDE may be impractical for moving sources.

Larger time-bandwidth products of the observed signals are required in order to make TDE feasible in environments with signal coherence loss. As discussed with respect to the CRB, only the observation time is controllable in passive applications, thus leading us to consider source motion models in Section 4 for use during long observation intervals.

We can extend the analysis of the $H=2$ sensor case to TDE with $H>2$ sensors following the approach of Weinstein, ${ }^{17}$ leading to the conclusion that pairwise TDE is essentially optimum for cases of interest with reasonable signal coherence between sensors. By pairwise TDE we mean that one sensor, say $H$, is identified as the reference, and only the $H-1$ time differences $D_{1 H}, D_{2 H}, \ldots, D_{H-1, H}$ are estimated. Under the conditions described below, these $H-1$ estimates are nearly as accurate for source localization as forming all pairs of TDEs $D_{g h}$ for all $g<h$. Weinstein's analysis ${ }^{17}$ is valid for moving as well as non-moving sources.

Extending (25) and (26) to $H>2$ sensors, let us assume equal $G_{s, h h} / G_{w}$ at all sensors $h=1, \ldots, H$ and equal coherence $\gamma_{s}$ between all sensor pairs, so that the $\operatorname{SNR}\left(\left|\gamma_{s}\right|\right)$ in (26) is equal for all sensor pairs. Then 


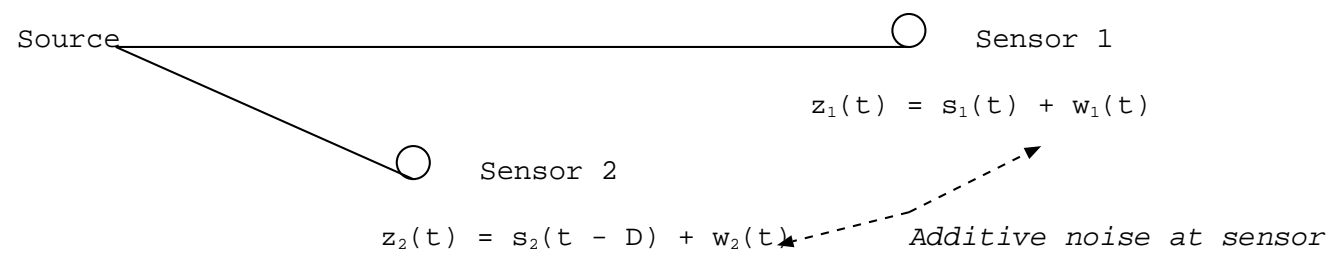

(a)

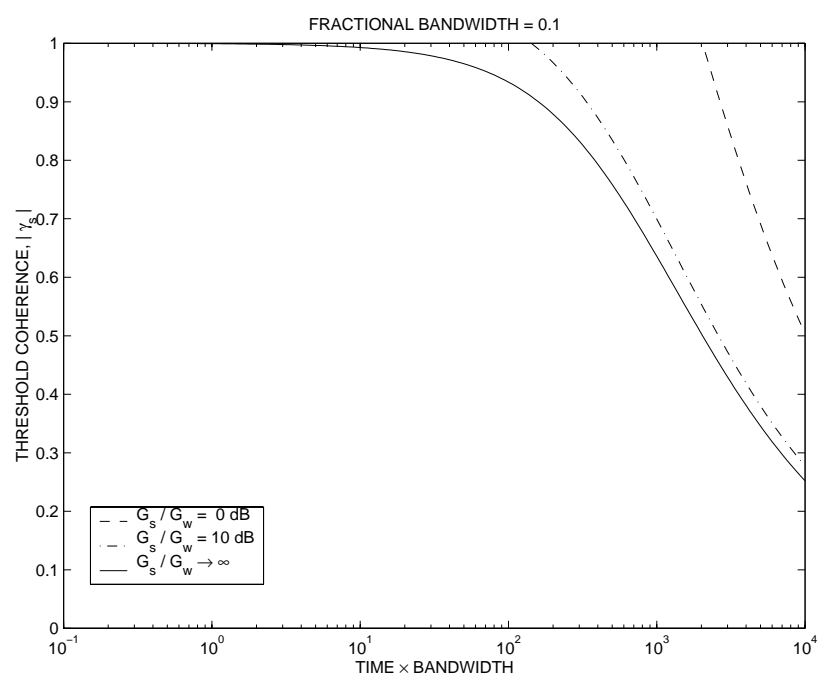

(b)

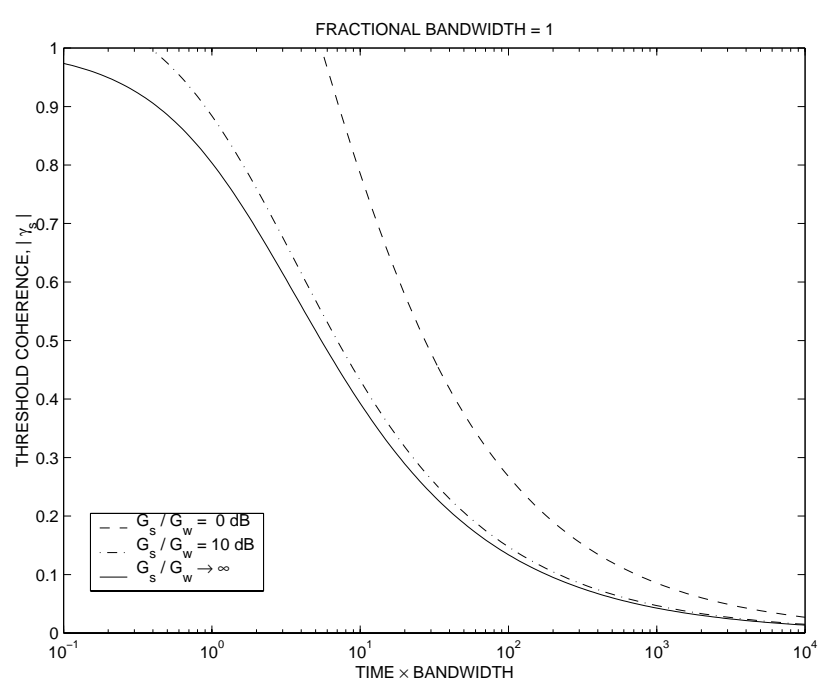

(d)

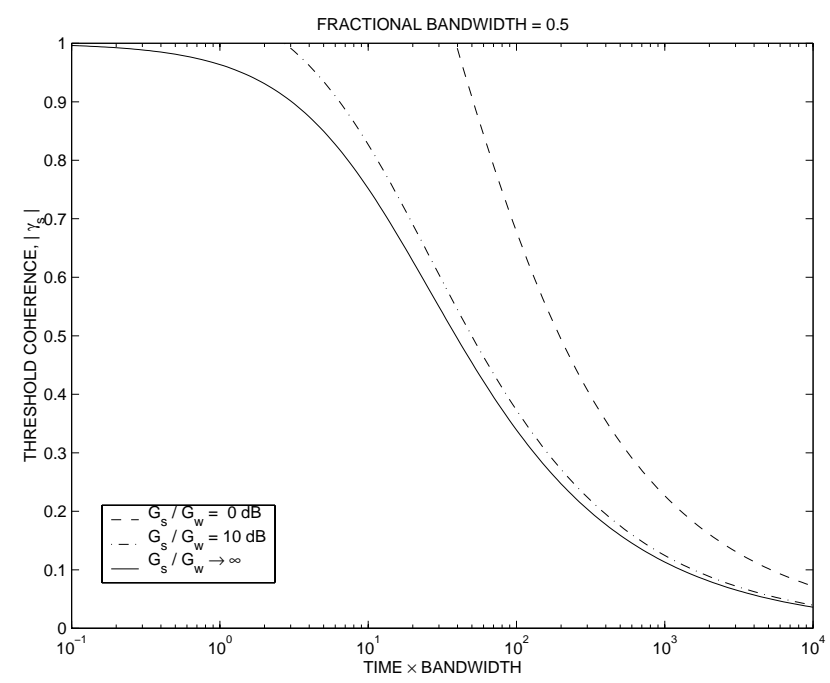

(c)

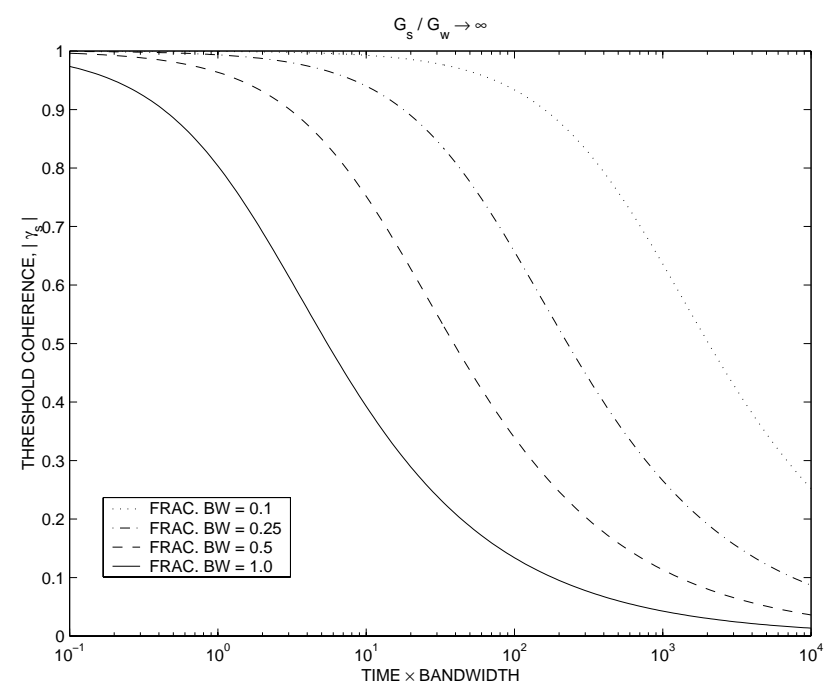

(e)

Figure 2. (a) Time-delay estimation (TDE) problem for a non-moving source with $H=2$ sensors. (b)-(e): Threshold coherence value from (28) versus time-bandwidth product $\left(\frac{\Delta \omega \cdot \mathcal{T}}{2 \pi}\right)$ and SNR $G_{s} / G_{w}$ for fractional bandwidth values $\left(\frac{\Delta \omega}{\omega_{0}}\right)$ (b) 0.1 , (c) 0.5 , and (d) 1.0. In (e), the high SNR curves $G_{s} / G_{w} \rightarrow \infty$ are superimposed for several values of fractional bandwidth. 
as long as $H \cdot \operatorname{SNR}\left(\left|\gamma_{s}\right|\right) \gg 1$, we can show that forming all TDE pairs $D_{g h}$ potentially improves the source localization variance relative to pairwise processing by the factor

$$
V=\frac{H\left(1+2 \cdot \frac{\gamma_{s}}{1-\gamma_{s}}\right)}{2\left(1+H \cdot \frac{\gamma_{s}}{1-\gamma_{s}}\right)} .
$$

Clearly $V \rightarrow 1$ as $\gamma_{s} \rightarrow 1$, and $V<(3 H) /[2(1+H)]<1.5$ for $\gamma_{s}>0.5$. Therefore the potential accuracy gain from processing all sensor pairs is negligible when the coherence exceeds the threshold values that are typically required for TDE.

This result suggests strategies with moderate communication bandwidth that potentially achieve nearly optimum localization performance. The reference sensor, $H$, sends its raw data to all other sensors. Those sensors $h=1, \ldots, H-1$, locally estimate the time differences $D_{1, H}, \ldots, D_{H-1, H}$, and these estimates are passed to the fusion center for localization processing with the bearing estimates $\phi_{1}, \ldots, \phi_{H}$. A modified scheme with more communication bandwidth but more centralized processing is for all $H$ sensors to communicate their data to the fusion center, with TDE performed at the fusion center.

\section{DATA MODEL FOR A MOVING SOURCE}

Our objective in this paper is to quantify scenarios in which jointly processing data from widely-spaced sensors has the potential for improved source localization accuracy, compared with incoherent triangulation/tracking of bearing estimates. We established in Section 2 that the potential for improved accuracy depends directly on TDE between the sensors. Then we showed in Section 3 that TDE with partially-coherent signals is feasible only with an increased time-bandwidth product of the sensor signals. This leads to a constraint on the minimum observation time, $\mathcal{T}$, in passive applications where the signal bandwidth is fixed. If the source is moving, then approximating it as non-moving becomes poorer as $\mathcal{T}$ increases, so modeling the source motion becomes more important.

Approximate bounds are known ${ }^{18,19}$ that specify conditions of validity for non-moving and moving source models. Let us consider $H=2$ sensors with Doppler values $\alpha_{2}>\alpha_{1}$ (see (43) for the definitions of $\alpha_{1}, \alpha_{2}$ ). If $f_{\max }(\mathrm{Hz})$ is the maximum signal frequency that is processed, then TDE estimation accuracy is not seriously affected by ignoring source motion, as long as the time interval $\mathcal{T}$ satisfies

$$
\mathcal{T} \ll \frac{1}{f_{\max }\left(\frac{\alpha_{2}}{\alpha_{1}}-1\right)} .
$$

Taking typical parameters for ground vehicles in aeroacoustics, let us consider a vehicle moving at $5 \%$ the speed of sound $(15 \mathrm{~m} / \mathrm{sec})$, with radial motion that is in opposite directions at the two sensors. Then $\alpha_{2} / \alpha_{1}-1 \approx 0.1$ and (30) becomes $\mathcal{T} \ll 10 / f_{\max }$. For $f_{\max }=100 \mathrm{~Hz}$, the requirement is $\mathcal{T} \ll 0.1 \mathrm{sec}$, which according to the analysis in Section 2 yields insufficient time-bandwidth product for partially coherent signals that are typically encountered. Thus motion modeling and Doppler compensation are critical, even for aeroacoustic sources that move more slowly than in this example.

In this section, we extend the non-moving source model from Section 2 using first-order motion models (see (31),(32),(46)). The first-order motion models are simple and accurate over larger time intervals $\mathcal{T}$ compared with the non-moving source model. However, accurate modeling of more complex trajectories over longer time intervals requires higher-order polynomial models, with added complexity. The source position trajectory is modeled as a straight line with constant velocity over an interval of length $\mathcal{T}$,

$$
\begin{aligned}
& x_{s}(t)=x_{s, 0}+\dot{x}_{s} \cdot\left(t-t_{0}\right), \quad t_{0} \leq t \leq t_{0}+\mathcal{T} \\
& y_{s}(t)=y_{s, 0}+\dot{y}_{s} \cdot\left(t-t_{0}\right),
\end{aligned}
$$

so $\dot{x}_{s}, \dot{y}_{s}$ are the velocity components. The source trajectory parameter vector is

$$
\boldsymbol{\Theta}=\left[x_{s, 0}, \dot{x}_{s}, y_{s, 0}, \dot{y}_{s}\right]^{T},
$$


and the (time-varying) propagation time from the source to the sensors on array $h$ follows from (1) and (2):

$$
\begin{aligned}
\tau_{h}(t) & =\frac{d_{h}(t)}{c}=\frac{1}{c}\left[\left(x_{s}(t)-x_{h}\right)^{2}+\left(y_{s}(t)-y_{h}\right)^{2}\right]^{1 / 2} \\
\tau_{h n}(t) & \approx-\frac{1}{c}\left[\frac{x_{s}(t)-x_{h}}{d_{h}(t)} \Delta x_{h n}+\frac{y_{s}(t)-y_{h}}{d_{h}(t)} \Delta y_{h n}\right]=-\frac{1}{c}\left[\left(\cos \phi_{h}(t)\right) \Delta x_{h n}+\left(\sin \phi_{h}(t)\right) \Delta y_{h n}\right] .
\end{aligned}
$$

The bearing and bearing rate are related to the source motion parameters $\Theta$ as

$$
\begin{aligned}
& \phi_{h}(t)=\tan ^{-1}\left[\frac{y_{s}(t)-y_{h}}{x_{s}(t)-x_{h}}\right]=\tan ^{-1}\left[\frac{y_{s, 0}+\dot{y}_{s} \cdot\left(t-t_{0}\right)-y_{h}}{x_{s, 0}+\dot{x}_{s} \cdot\left(t-t_{0}\right)-x_{h}}\right] \\
& \dot{\phi}_{h}(t)=\frac{\dot{y}_{s} \cos \phi_{h}(t)-\dot{x}_{s} \sin \phi_{h}(t)}{d_{h}(t)} .
\end{aligned}
$$

The radial velocity of the source with respect to array $h$ is

$$
v_{r, h}(t)=\dot{x}_{s} \cos \phi_{h}(t)+\dot{y}_{s} \sin \phi_{h}(t) .
$$

We can insert (31) and (32) into (34) to obtain the following approximation for the propagation time to array $h$ :

$$
\begin{aligned}
\tau_{h}(t) & =\tau_{h}\left(t_{0}\right)\left[1+\frac{2 \cdot \cos \phi_{h}\left(t_{0}\right) \cdot \dot{x}_{s} \cdot\left(t-t_{0}\right)}{d_{h}\left(t_{0}\right)}+\frac{2 \cdot \sin \phi_{h}\left(t_{0}\right) \cdot \dot{y}_{s} \cdot\left(t-t_{0}\right)}{d_{h}\left(t_{0}\right)}\right]^{1 / 2} \\
& \approx \tau_{h}\left(t_{0}\right)+\frac{v_{r, h}\left(t_{0}\right)}{c} \cdot\left(t-t_{0}\right),
\end{aligned}
$$

where $d_{h}\left(t_{0}\right)$ and $v_{r, h}\left(t_{0}\right)$ are the source distance and radial velocity at the start of time interval $t=t_{0}$. The approximation (40) is valid as long as the total motion during the time interval $\mathcal{T}$ is much less than the range, i.e., $\left|2 \dot{x}_{s} \mathcal{T}\right| \ll d_{h}\left(t_{0}\right)$ and $\left|2 \dot{y}_{s} \mathcal{T}\right| \ll d_{h}\left(t_{0}\right)$.

Next we use the approximation (40) and model the received signal at the reference sensor on array $h$ as

$$
\begin{aligned}
s_{h}\left(t-\tau_{h}(t)\right) & =s_{h}\left[\left(1-\frac{v_{r, h}\left(t_{0}\right)}{c}\right) t-\tau_{h}\left(t_{0}\right)+\frac{v_{r, h}\left(t_{0}\right) t_{0}}{c}\right] \\
& =s_{h}\left(\alpha_{h} t-\tau_{h}\left(t_{0}\right)+\frac{v_{r, h}\left(t_{0}\right) t_{0}}{c}\right), \quad t_{0} \leq t \leq t_{0}+\mathcal{T},
\end{aligned}
$$

where

$$
\alpha_{h}=1-\frac{v_{r, h}\left(t_{0}\right)}{c}=1-\frac{1}{c}\left[\dot{x}_{s} \cos \phi_{h}\left(t_{0}\right)+\dot{y}_{s} \sin \phi_{h}\left(t_{0}\right)\right]
$$

is the Doppler compression and

$$
\tau_{h}\left(t_{0}\right)=\frac{d_{h}\left(t_{0}\right)}{c}=\frac{1}{c}\left[\left(x_{s, 0}-x_{h}\right)^{2}+\left(y_{s, 0}-y_{h}\right)^{2}\right]^{1 / 2}
$$

is the propagation delay at the initial time $t=t_{0}$. Without loss of generality, we set $t_{0}=0$, so the received signal at sensor $n$ on array $h$ is

$$
s_{h}\left(\alpha_{h} t-\tau_{h}(0)-\tau_{h n}(t)\right),
$$

which is the extension of the signal component of (8) to the moving source case. Note from (2) that $\tau_{h n}(t)$ depends on the source location only through the time-varying bearing $\phi_{h}(t)$, which we approximate with a first-order model

$$
\phi_{h}(t) \approx \phi_{h}\left(t_{0}\right)+\dot{\phi}\left(t_{0}\right) \cdot\left(t-t_{0}\right), \quad t_{0} \leq t \leq t_{0}+\mathcal{T} .
$$

For a single array $h$, the Doppler compression $\alpha_{h}$ and time delay $\tau_{h}\left(t_{0}\right)$ have negligible effect on estimation of the the intra-array delays $\tau_{h n}(t)$, since $\alpha_{h}$ and $\tau_{h}\left(t_{0}\right)$ are identical for each $n=1, \ldots, N_{h}$. Thus each array can be processed separately to estimate the bearings $\phi_{1}\left(t_{0}\right), \ldots, \phi_{H}\left(t_{0}\right)$ and bearing rates $\dot{\phi}_{1}\left(t_{0}\right), \ldots, \dot{\phi}_{H}\left(t_{0}\right)$, and these 
can be "triangulated" via (36) and (37) to estimate the source motion parameters $\boldsymbol{\Theta}$ in (33). An algorithm ${ }^{20}$ for estimating $\phi_{h}\left(t_{0}\right)$ and $\dot{\phi}_{h}\left(t_{0}\right)$ is described in Section 5.

Let us consider the signals received at the reference sensors at each array, so $\tau_{h n}(t)=0$ in (45):

$$
s_{1}\left[\left(1-\frac{v_{r, 1}\left(t_{0}\right)}{c}\right) t-\tau_{1}\left(t_{0}\right)\right], \ldots, s_{H}\left[\left(1-\frac{v_{r, H}\left(t_{0}\right)}{c}\right) t-\tau_{H}\left(t_{0}\right)\right] .
$$

Our modeling assumptions imply that each signal $s_{h}\left[\left(1-\frac{v_{r, h}\left(t_{0}\right)}{c}\right) t-\tau_{h}\left(t_{0}\right)\right]$ is a wide-sense stationary Gaussian random process. However, for two arrays $g, h$ with unequal Doppler $v_{r, g}\left(t_{0}\right) \neq v_{r, h}\left(t_{0}\right)$, the signals at arrays $g, h$ are not jointly wide-sense stationary, ${ }^{18,21}$ complicating the analytical description and the CRB performance analysis. The jointly nonstationary sensor signals generally are not characterized by a cross-spectral density matrix, so the CRB is not the inverse of a FIM of the form (15). An approximate CRB analysis for TDE with jointly nonstationary signals as in (47) was derived by Knapp and Carter. ${ }^{18}$ The CRB analysis was rigorously justified by Schultheiss and Weinstein, ${ }^{21}$ and they extended the results to CRBs on differential Doppler. A clever transformation is used so that the jointly nonstationary signals in (47) are locally modeled by a CSD of the form (14), and Schultheiss and Weinstein $\operatorname{show}^{21}$ that the representation is accurate for CRB analysis.

Formulating the results of Schulthiess and Weinstein ${ }^{21}$ for the case of partially coherent signals*, we make the following observations for $H=2$ arrays. The results are valid for large observation time $(\mathcal{T}$ much larger than the coherence time of the signals and noise). The TDE is $D_{12}=\tau_{1}\left(t_{0}\right)-\tau_{2}\left(t_{0}\right)$ and the differential Doppler is $\Delta v_{12}=v_{r, 1}\left(t_{0}\right)-v_{r, 2}\left(t_{0}\right)$.

- Estimation of TDE and differential Doppler are decoupled, so the CRB on $D_{12}$ is given by (21), which is identical to the non-moving source case.

- The threshold coherence analysis for TDE in (28) and Figure 2 extends to the moving source case. In the best case that Doppler effects are perfectly estimated and compensated, the TDE problem that remains is identical to the non-moving source case. Doppler estimation is less demanding in terms of time-bandwidth product compared with TDE. Indeed, Doppler estimation is possible with sinusoidal signals that have negligible bandwidth. ${ }^{21}$

The CRB on differential Doppler, ${ }^{21}$ modified for partially-coherent signals, is

$$
\operatorname{CRB}\left(\Delta v_{12}\right)=\frac{24 \pi}{\mathcal{T}}\left(\frac{c}{\mathcal{T}}\right)^{2}\left[2 \int_{0}^{\omega_{s}} \omega^{2} \operatorname{SNR}\left(\left|\gamma_{s, 12}(\omega)\right|\right) d \omega\right]^{-1}
$$

Note that (48) is a scalar multiple of the CRB on TDE in (21). The CRB on differential Doppler may be achievable in scenarios where the time-bandwidth product is insufficient for TDE.

Interestingly, differential Doppler provides sufficient information for source localization, even without TDE, as long as five or more sensors are available. ${ }^{21}$ Thus the source motion may be exploited in scenarios where TDE is not feasible, such as narrowband signals.

- We discussed TDE with $H>2$ sensors in Section 2, concluding that pairwise processing of TDEs $D_{1 H}, \ldots, D_{H-1, H}$ with a reference sensor $H$ is nearly optimum for scenarios of interest (see (29)). A similar result holds for differential Doppler estimation, ${ }^{21}$ where pairwise estimation of $\Delta v_{1 H}, \ldots, \Delta v_{H-1, H}$ is nearly as accurate as estimation of all pairs $\Delta v_{g h}$, as long as $H \cdot \operatorname{SNR}\left(\left|\gamma_{s}\right|\right) \gg 1$.

\section{AN ALGORITHM}

The parameters that can be directly estimated from the sensor data are the bearings $\phi_{1}\left(t_{0}\right), \ldots, \phi_{H}\left(t_{0}\right)$, bearing rates $\dot{\phi}_{1}\left(t_{0}\right), \ldots, \dot{\phi}_{H}\left(t_{0}\right)$, pairwise time differences $D_{1 H}=\tau_{1}\left(t_{0}\right)-\tau_{H}\left(t_{0}\right), \ldots, D_{H-1, H}=\tau_{H-1}\left(t_{0}\right)-\tau_{H}\left(t_{0}\right)$, and differential Doppler $\Delta v_{1 H}=v_{r, 1}\left(t_{0}\right)-v_{r, H}\left(t_{0}\right), \ldots, \Delta v_{H-1, H}=v_{r, H-1}\left(t_{0}\right)-v_{r, H}\left(t_{0}\right)$. Equations (36), (37), (43),

${ }^{*}$ The signal coherence between the signals at arrays $g$ and $h$ in (47) is defined assuming perfect compensation of the Doppler compression $\alpha_{g}, \alpha_{h}$, thus yielding the definition in (7). 
(44) define the nonlinear relations that "triangulate" these parameters and relate them to the source motion parameters $\boldsymbol{\Theta}=\left[x_{s, 0}, \dot{x}_{s}, y_{s, 0}, \dot{y}_{s}\right]^{T}$.

A distributed processing algorithm is outlined below.

1. Use the local polynomial approximation (LPA) beamformer ${ }^{20}$ at each array to estimate the bearings and bearing rates. The LPA beamformer in $^{20}$ is formulated for narrowband processing, and it is a generalization of the classical beamformer to moving sources. We extend it in a straightforward way to wideband signals by incoherently averaging the LPA beampatterns at different frequencies.

2. Solve (36), (37) to obtain initial estimates of the source motion parameters $\boldsymbol{\Theta}$. These estimates correspond to incoherent triangulation of the bearings and bearing rates from individual arrays.

3. Estimate the Doppler compression factors $\alpha_{1}, \ldots, \alpha_{H}$, compensate for Doppler, and test whether the signals at distinct arrays have sufficient coherence, fractional bandwidth, and time-bandwidth product to enable TDE between arrays (see Section 2 for the conditions).

4. If the conditions are not met, then incoherent triangulation of the bearings and bearing rates is nearly optimum, and further joint processing is not informative.

5. If the conditions are met, then identify a reference array $H$ (the array with maximum SNR) and estimate the time differences $D_{1 H}, \ldots, D_{H-1, H}$ and differential Dopplers $\Delta v_{1 H}, \ldots, \Delta v_{H-1, H}$. The maximum likelihood solution involves wideband ambiguity function search over Doppler and TDE, ${ }^{18}$ but computationally simpler alternatives have been investigated. ${ }^{22}$

6. A suboptimum procedure is to avoid the joint Doppler and TDE estimation in the preceding step, and instead use the initial Doppler estimates from steps 1 and 2 and perform TDE after approximate Doppler compensation. With this approach, triangulation of the TDEs via (44) will improve the estimates of $x_{s, 0}$ and $y_{s, 0}$ only (and not the source velocity $\dot{x}_{s}, \dot{y}_{s}$ ).

7. If multiple sources are present, then the LPA beamformer in step 1 may be used to separate the source signals at each array prior to Doppler/TDE estimation.

We have presented an example of the LPA beamformer in steps 1 and 7 for a two-source scenario based on measured aeroacoustic data elsewhere, ${ }^{16}$ and we have also presented examples of TDE with Doppler compensation (step 5$).^{7}$

\section{CONCLUDING REMARKS}

The potential gain in source localization accuracy when data from distributed arrays is processed jointly and coherently is quantified by the CRBs presented in this paper. The amount of improvement and the feasibility of achieving the improvement depend critically on the scenario, which is characterized by the coherence between source signals arriving at distributed sensors, the signal bandwidth and spectrum shape (wideband vs. harmonic), the observation time for coherent processing, the noise level, the source motion parameters (velocity, complexity of maneuvers), and the number of sources. In feasible scenarios in which the time-bandwidth product is large enough to enable TDE, we proposed an algorithm that requires moderate communication bandwidth between sensors. The processing involves estimation of bearing and bearing rate at individual arrays, and estimation of time delay and differential Doppler between pairs of arrays. 


\section{REFERENCES}

1. R.R. Tenney and J.R. Delaney, "A distributed aeroacoustic tracking algorithm," Proc. American Control Conf., pp. 1440-1450, June 1984.

2. Y. Bar-Shalom and X.-R. Li, Multitarget-Multisensor Tracking: Principles and Techniques, YBS, 1995.

3. A. Farina, "Target tracking with bearings-only measurements," Signal Processing, vol. 78, pp. 61-78, 1999.

4. B. Ristic, S. Arulampalam, C. Musso, "The influence of communication bandwidth on target tracking with angle only measurements from two platforms," Signal Processing, vol. 81, pp. 1801-1811, 2001.

5. L.M. Kaplan, P. Molnar, Q. Le, "Bearings-only target localization for an acoustical unattended ground sensor network," Proc. SPIE AeroSense, Orlando, Florida, April 2001.

6. R.J. Kozick and B.M. Sadler, "Source Localization with Distributed Sensor Arrays and Partial Spatial Coherence," SPIE 2000 AeroSense Symp., Orlando, FL, pp. 142-153, April 24-28, 2000.

7. R.J. Kozick and B.M. Sadler, "Near-Field Localization of Acoustic Sources with Imperfect Spatial Coherence, Distributed Processing, and Low Communication Bandwidth," SPIE 2001 AeroSense Symp., Vol. 4393, pp. 52-63, Orlando, FL, April, 2001.

8. B. Friedlander, "On the Cramer-Rao Bound for Time Delay and Doppler Estimation," IEEE Trans. on Info. Theory, vol. IT-30, no. 3, pp. 575-580, May 1984.

9. P. Whittle, "The analysis of multiple stationary time series," J. Royal Statist. Soc., vol. 15, pp. 125-139, 1953.

10. S.M. Kay, Fundamentals of Statistical Signal Processing: Estimation Theory, Prentice-Hall, 1993.

11. G.C. Carter (ed.), Coherence and Time Delay Estimation (Selected Reprint Volume), IEEE Press, 1993.

12. W.F. Walker and G.E. Trahey, "A fundamental limit on delay estimation using partially correlated speckle signals," IEEE Trans. on Ultrasonics, Ferroelectrics, and Frequency Control, vol. 42, no. 2, pp. 301-308, March 1995.

13. I. Cespedes, J. Ophir, S.K. Alam, "The combined effect of signal decorrelation and random noise on the variance of time delay estimation," IEEE Trans. on Ultrasonics, Ferroelectrics, and Frequency Control, vol. 44, no. 1, pp. 220-225, Jan. 1997.

14. A.J. Weiss and E. Weinstein, "Fundamental limitations in passive time delay estimation - part 1: narrowband systems," IEEE Trans. Acoust., Speech, Sig. Proc., vol. ASSP-31, no. 2, pp. 472-485, April 1983.

15. E. Weinstein and A.J. Weiss, "Fundamental limitations in passive time delay estimation - part 2: wideband systems," IEEE Trans. Acoust., Speech, Sig. Proc., vol. ASSP-32, no. 5, pp. 1064-1077, Oct. 1984.

16. R.J. Kozick and B.M. Sadler, "Issues and Algorithms for Tracking Multiple Sources with a Network of Sensors," Proc. 2001 Meeting of the MSS Specialty Group on Battlefield Acoustics and Seismics, Laurel, MD, October 23-26, 2001.

17. E. Weinstein, "Decentralization of the Gaussian maximum likelihood estimator and its applications to passive array processing," IEEE Trans. Acoust., Speech, Sig. Proc., vol. ASSP-29, no. 5, pp. 945-951, October 1981.

18. C.H. Knapp and G.C. Carter, "Estimation of time delay in the presence of source or receiver motion," $J$. Acoust. Soc. Am., vol. 61, no. 6, pp. 1545-1549, June 1977.

19. W.B. Adams, J.P. Kuhn, W.P. Whyland, "Correlator compensation requirements for passive time-delay estimation with moving source or receivers," IEEE Trans. Acoust., Speech, Signal Processing, vol. ASSP-28, no. 2, pp. 158-168, April 1980.

20. V. Katkovnik and A.B. Gershman, "A local polynomial approximation based beamforming for source localization and tracking in nonstationary environments," IEEE Signal Processing Letters, vol. 7, no. 1, pp. 3-5, Jan. 2000.

21. P.M. Schultheiss and E. Weinstein, "Estimation of differential Doppler shifts," J. Acoust. Soc. Am., vol. 66, no. 5, pp. 1412-1419, Nov. 1979.

22. J.W. Betz, "Comparison of the deskewed short-time correlator and the maximum likelihood correlator," IEEE Trans. Acoust., Speech, Signal Processing, vol. ASSP-32, no. 2, pp. 285-294, April 1984. 